\title{
Staphylococcus aureus, Toll-like receptors, superantigens, and their derivatives
}

\author{
Friedrich C. Luft
}

Published online: 12 August 2012

(C) Springer-Verlag 2012

Some patients just cannot get out of my mind, irrespective of how long ago it was. A young woman was admitted to my intensive care unit in 1979. She had a body temperature $>39^{\circ} \mathrm{C}$, a systolic blood pressure $<90 \mathrm{mmHg}$, and a remarkable diffuse erythroderma that covered her entire body. A vaginal discharge was present and a large tampon had been removed in the emergency department. The patient was confused and could not answer our questions. Her hands and feet showed signs of desquamation. Renal failure, elevated liver enzymes, marked thrombocytopenia, metabolic acidosis, and hypoxemia were revealed by the laboratory. Volume expansion, vasopressors, methicillin, and vancomycin were given and she appeared to be stabilized. We knew that Staphylococcus aureus was responsible. However, thereafter she again lapsed into shock and died several hours later. We were aware of the term "toxic shock syndrome" and improper tampon use throughout menstruation was later implicated in the condition [1]. Thirty-three years later, I may finally find out how this deadly disease worked and why our therapeutic attempts failed so dismally.

Superantigens are a class of antigens that cause nonspecific polyclonal $\mathrm{T}$ cell activation and massive cytokine release. Pathogenic viruses, mycoplasma, and bacteria can produce superantigens as a defense mechanism against the host immune system. Superantigens can activate up to $20 \%$ of the body's T cells. Superantigens are produced intracellularly by bacteria and are released upon infection as extracellular toxins. S. aureus produces various superantigens [2].

In flies, Toll encodes proteins important to establishing the dorsal-ventral axis during embryogenesis. Later, Toll

\section{F. C. Luft $(\bowtie)$}

Experimental and Clinical Research Center,

Max-Delbrück Center and Charité Medical Faculty,

Lindenbergerweg 80 ,

13125 Berlin, Germany

e-mail: luft@charite.de was found to have a role in the fly's immunity to fungal infections [3]. Toll-like receptors (TLRs) are pattern recognition receptors that recognize broadly shared molecules of pathogens. TLR together with the interleukin-1 (IL-1) receptors form a receptor superfamily. Members have a socalled Toll-IL-1 receptor domain in common. Myeloid differentiation primary response gene-88 (Myd88) is an adaptor protein that is commonly used by TLR to activate the transcription nuclear factor kappa B. TLR-2 is a membrane protein, a receptor, which is expressed on the surface of certain cells and recognizes foreign substances and passes on appropriate signals to the cells of the immune system [4]. As a membrane surface receptor, TLR-2 recognizes many bacterial, fungal, viral, and certain endogenous substances (Fig. 1). TLR-2 dimerizes with TLR-1 and TLR-6. There are other participating molecules, such as CD14 and CD36. TLR-2 is pivotal for host protection against $S$. aureus.

Bacteria are evidently not stupid and $S$. aureus has developed an array of defensive substances and evasive maneuvers $[5,6]$. The staphylokinase inhibits the bactericidal effects of human defensins and activates human plasminogen. Staphylococci produce aureolysin that inactivates LL-37, a human bactericidal peptide. Staphylococci also produce muramic acid that confers lysozyme resistance. Additional mechanisms make staphylococci resistant to reactive oxygen species and enable them to evade complement. Staphylococcal protein A is well-known for its capacity to bind the Fe part of IgG. Through this binding, protein A blocks $\mathrm{Fc}$ receptor-mediated phagocytosis and also complement activation via $\mathrm{C} 1 \mathrm{q}$. Protein $\mathrm{A}$ has turned out to be a convenient laboratory and clinical tool. S. aureus also produces a host of superantigen-like proteins (SSL) that contribute to the immune-evasive properties of these virulent bacteria. In this issue, Bardoel et al. [7] show us how staphylococcal SSL3 specifically binds and inhibits TLR-2 activation in neutrophils and monocytes. They show that 
Fig. 1 Signaling pathways mediated by Toll-like receptor 2 and partners. $S$. aureus offers potential interference by a superantigen-like 3 molecule. As a result, innate immunity is inhibited at the TLR-2 step and the bacteria are "protected"

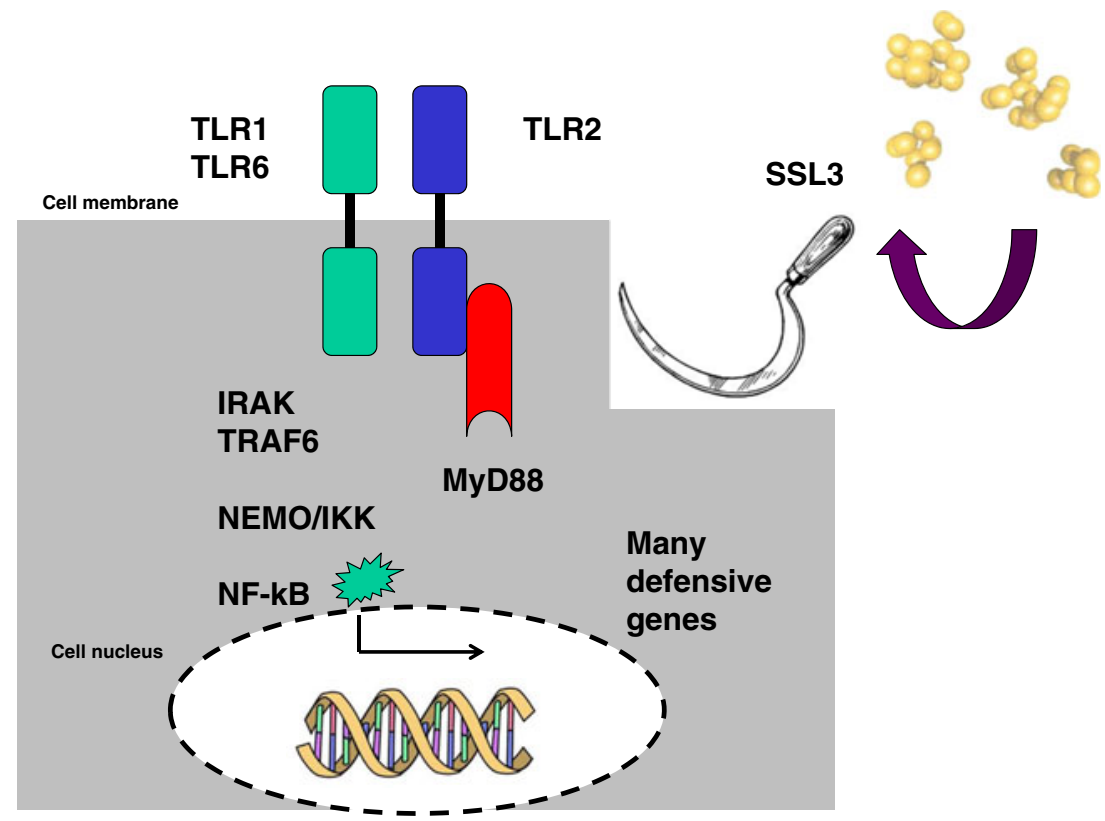

SSL3 specifically targets TLR-2 and that the binding to TLR-2 is in part sialic acid-dependent. SSL3 blocked the release of IL-8 in a cell culture system. IL-8 signals through serpentine receptors (CXCR1 and CXCR2) and is a major mediator of the inflammatory response necessary to induce chemotaxis. The authors' findings suggest that SSL3 is a unique TLR-2 inhibitor and largely eliminates neutrophils and monocytes from conducting innate immunity to protect hosts from staphylococcal infections. The data do not only give insight into another mechanism of protection for our wily foe, but also have more far reaching implications. There is much missing in terms of information. The authors draw attention to about 14 SSL proteins. Some information is known; however, most structural details are not. Thus, the mechanism of how SSL3 inhibits TLR-2 remains undefined.

We now have fairly detailed insight into the toxic shock syndrome. Staphylococcal superantigens unquestionably cause the syndrome $[8,9]$. The tampon-associated form that our patient had involves the presence of increased oxygen in the normally quite anaerobic vaginal epithelium. The superantigen, toxic shock syndrome toxin-1 (TSST-1), can penetrate this fragile epithelium and cause the release of IL-1, IL2 , tumor necrosis factor, and other mediators. TSST-1 binds primarily to the alpha chain of class II MHC on T cells and antigen-presenting cells. The subsequent, almost generalized, $\mathrm{T}$ cell activation results in massive cytokine release. Knowledge on SSL molecules, such as SSL3 that block innate defenses against staphylococci, shed more light on the lethality of the syndrome and the susceptibility to any staphylococcal infection. Indeed, the Center for Disease Control in the USA has determined that $S$. aureus is the leading cause of serious and fatal bacterial infection in the USA. The superantigen and superantigen-like molecules raise fundamental questions. Apparently, the role of both is an evasive maneuver of bacteria directed against innate and acquired immunity. However, if the host suddenly dies, the invader has no immediate advantage. No symbiotic relationship regarding these mechanisms has evidently as yet developed.

Microbial infections are also related to atherosclerosis and cardiovascular diseases. TLR plays a role in this process. TLR-2 has a large ligand repertoire that includes bacterialderived exogenous and possibly host-derived endogenous ligands. Mice deficient in the low-density lipoprotein receptor develop severe atherosclerosis when exposed to a high-fat diet. When the mice are also exposed to a TLR-2 agonist, the disease is substantially worsened further [10]. Furthermore, when these mice are bred into a strain deficient in TLR-2, a double knock-out can be generated. The resultant mice are substantially protected from the TLR-2 agonist. The same was the case in mice that solely lacked bone marrowderived cells expressing TLR-2. The studies support the concept that chronic or recurrent microbial infections may contribute to atherosclerotic disease. Additionally, the presence of host-derived endogenous TLR-2 agonists could aggravate atherosclerosis. However, perhaps SSL3 could be developed therapeutically. Since SSL3 clearly interferes with TLR-2 signaling, perhaps a derivative of the material could have a future as a cardiovascular drug.

Respectfully, Friedrich C. Luft 


\section{References}

1. Shands KN, Schmid GP, Dan BB, Blum D, Guidotti RJ, Hargrett NT, Anderson RL, Hill DL, Broome CV, Band JD et al (1980) Toxic-shock syndrome in menstruating women: association with tampon use and Staphylococcus aureus and clinical features in 52 cases. N Engl J Med 303:1436-1442

2. Llewelyn M, Cohen J (2002) Superantigens: microbial agents that corrupt immunity. Lancet Invest Dis 3:156-162

3. Hoffmann JA (2003) The immune response of Drosophila. Nature 426:33-38

4. Kirschning CJ, Schumann RR (2002) TLR2: cellular sensor for microbial and endogenous molecular patterns. Curr Top Microbiol Immunol 270:121-144

5. Rooijakkers SH, van Kessel KP, van Strijp JA (2005) Staphylococcal innate immune evasion. Trends Microbiol 13:596-601
6. Kim HK, Thammavongsa V, Schneewind O, Missiakas D (2012) Recurrent infections and immune evasion strategies of Staphylococcus aureus. Curr Opin Microbiol 15:92-99

7. Bardoel BW, Vos R, Bouman T, Aerts PC, Bestebroer J, Huizinga EG, Brondijk TH, van Strijp JA, de Haas CJ (2012) Evasion of Toll-like receptor 2 activation by staphylococcal superantigen-like protein 3. J Mol Med (Berl) DOI: 10.1007/ s00109-012-0926-8

8. Brosnahan AJ, Schlievert PM (2011) Gram-positive bacterial superantigen outside-in signaling causes toxic shock syndrome. FEBS J 278:4649-4667

9. Silversides JA, Lappin E, Ferguson AJ (2010) Staphylococcal toxic shock syndrome: mechanisms and management. Curr Infect Dis Rep 12:392-400

10. Mullick AE, Tobias PS, Curtiss LK (2005) Modulation of atherosclerosis in mice by Toll-like receptor 2. J Clin Invest 115:31493156 